\section{Emission Spectroscopy with Infra-Red Double-Beam Spectrophotometers}

Some time ago, K. A. Fischer and G. Brandes ${ }^{1}$ demonstrated that it is possible to obtain useful emission spectroscopic data with an infra-red doublebeam spectrophotometer, if the sample placed in the beam path of the spectrophotometer is warmed up with a closed sample beam shutter. Instead of the quotient of the radiation intensity passed by the object and of the unattenuated radiation intensity, the instrument now scans the quotient of the inherent radiation intensity of the object and of the reference beam intensity.

The sensitivity of this method can be increased 100 times, if the intensity of the reference beam is attenuated by means of a Venetian shutter by the same factor. Thus it is possible to record also the relatively weak emission maxima of moderately heated gases.

Fig. 1 shows the emission spectrum of ammonia, heated up to $160^{\circ} \mathrm{C}$., between 9 and $12 \mu$, recorded with a ten-fold reference beam attenuation. The thickness of the ammonia sample was $5 \mathrm{~cm}$. The spectrum was recorded with a Model 21 Infra-red Spectrophotometer of Bodenseewerk Perkin-Elmer and Co., GmbH. The attenuation of the reference beam was effected by a reference beam attenuator designed as an accessory to this instrument, the object being warmed up in a heatable gas cell, which likewise constitutes a usual spectrophotometer accessory though serving other purposes otherwise. The spectral dispersion was effected with a sodium chloride prism; the slit-width was controlled with the 'slit programme 800 '. This slit programme provides a slit-width of $55 \mu$ at a wave-length of $10 \mu$. Because of the low energy striking the thermocouple, the instrument was operated with maximum amplification, maximum time constant and a scanning speed of $5 \times 10^{-4} \mathrm{\mu} / \mathrm{sec}$. The spectrum distinctly shows the emission maxima assigned to the absorption bands of the ammonia. Fig. 2 shows the absorption spectrum of the same sample, which was scanned with

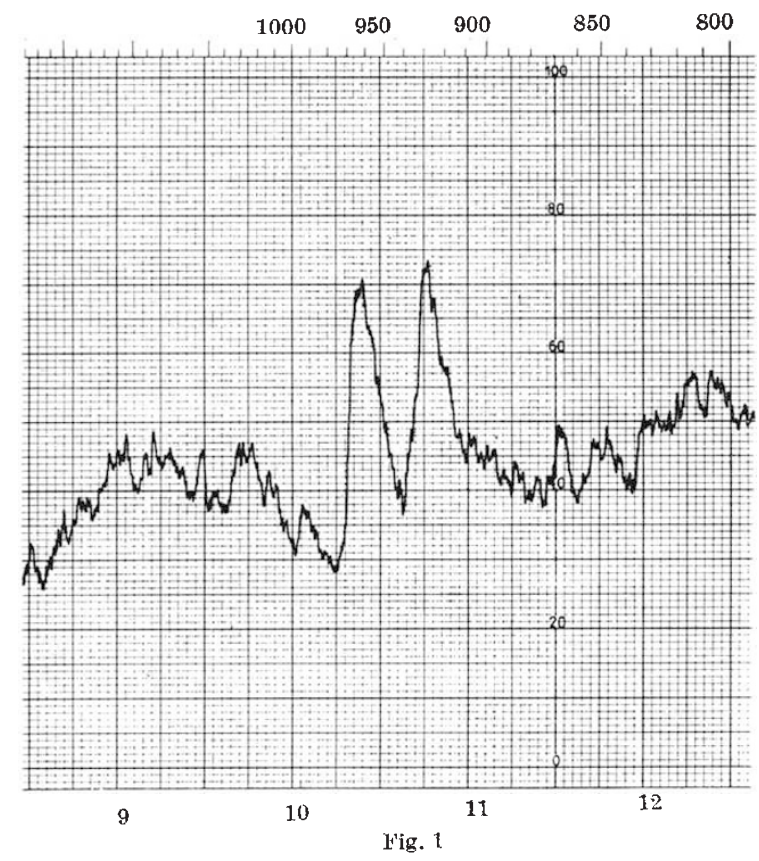

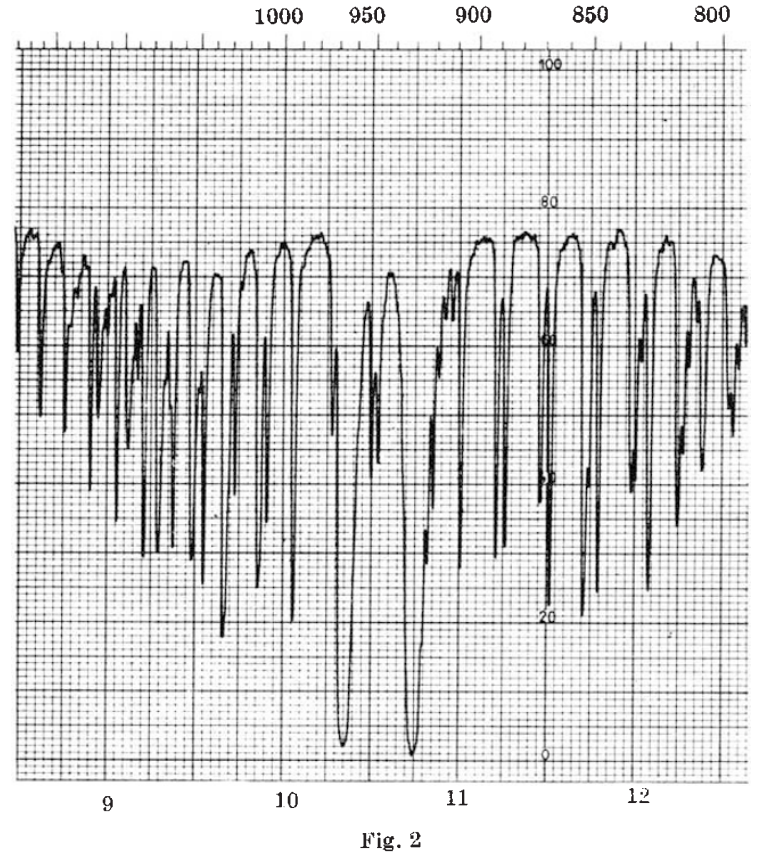

the same slit programme, however, without reference beam attenuation. It becomes obvious that the frequency conformity of the emission and absorption maxima has been realized quite well. The intensity ratios between the individual maxima are, however, considerably changed.

\section{J. BRAUNBFCK}

Bodenseewerk Perkin-Elmer and Co., GmbH.

Überlingen - Bodensee.

${ }^{1}$ Fischer, K. A., and Brandes, (.., Naturwiss., 43, 223 (1956).

\section{Rotational Analysis of the $C^{2} \Pi-X^{2} \Sigma^{+}$ System of $\mathrm{CaCl}$}

ALthougr many of the electronie band-systems of the monohalides of Group II $A$ are readily observed in emission and absorption, little is known about the properties of these molecules, other than their force constants and the positions of their lower-lying electronic energy states. Only one complete rotational analysis, of the system $A^{2} \Pi_{1}-X^{2} \Sigma^{+}$in BeF has yet been given ${ }^{1}$.

In many cases, the rotational structure is, through the near equality of $B$-values, too close to be resolved, but new photographs on a $6 \cdot 65-\mathrm{m}$. grating spectrograph suggest that analyses of some systems will be possible. One such system is $C^{2} \Pi-X^{2} \Sigma^{+}$in $\mathrm{CaCl}$, which may conveniently be observed in absorption. We have been able to analyse the 0,0 band, and the shorter wave-length sub-bands of the 1,0 and 0,1 bands. The upper state is sufficiently close to Hund's case $a$ for the two sub-bands to be treated independently to give effective values of $B^{\prime}$ and $D^{\prime}$. The $\Lambda$-type doubling, which is negligibly small in the shorter wave-length sub-bands, but observable in the longer wave-length components, shows that the $C$ state is " $\Pi_{\text {reg., }}$ presumably $\ldots(w \pi)^{4}(v \pi),{ }^{2} \Pi_{r}$. An earlier, incomplete analysis ${ }^{2}$ of this system is incorrect. The constants obtained in the present analysis are summarized in Table 1. 